\title{
Histochemical demonstration of acetylcholinesterase in neuroblastoma
}

\author{
S VARIEND, MA LOUGHLIN \\ From the Department of Pathology, Children's Hospital, Western Bank, Sheffield S10 2TH
}

SUMMARY The presence of acetylcholinesterase in the tumour cells of neuroblastoma has been shown by enzyme histochemistry. For comparison, some other tumours likely to be found in children and commonly presenting histologically as small cell tumours have also been studied. Acetylcholinesterase activity was seen in rhabdomyosarcoma, but, compared with neuroblastoma, the activity was focal and sparse. One Ewing's tumour and a lymphoblastic lymphoma were negative for the enzyme reaction.

Some of the ultrastructual features of neuroblastoma are correlated with the presence of this enzyme. Acetylcholinesterase enzyme histochemistry may provide a useful adjunct in the distinction of neuroblastoma from other small cell tumours.

Choice of treatment and prognosis in childhood malignancy are determined largely by tumour cell type. Childhood tumours often present histologically as small cell tumours, which may be difficult to resolve by conventional microscopy alone. Rosette formation, neurofibrillary material, and early ganglionic differentiation are light microscopic features which favour a diagnosis of neuroblastoma. These criteria are not always present, however, and, consequently, neuroblastoma may be difficult to distinguish from Ewing's sarcoma, rhabdomyosarcoma, and lymphoma. Electron microscopy,' immunocytochemistry for neurone specific enolase, ${ }^{2}$ and induced catecholamine fluorescence, ${ }^{3}$ as well as monoclonal antibodies, ${ }^{4}$ have all been recommended to aid histological diagnosis, but many of these procedures are lengthy, are not without technical problems, and are often not specific for neuroblastoma.

The concept of "one nerve cell, one neurotransmitter" has been challenged recently. Morphological and pharmacological evidence exists in animals that distinction between neurone groups with regard to their content of neurotransmitter is not clear cut. ${ }^{5}$ Until quite recently, catecholamine secretion had generally been assumed to be the only biochemical expression of neuroblastoma. But several studies on neuroblastoma have now shown biochemically the presence of an acetylcholine synthesising enzyme, choline acetyltransferase,$^{6-8}$ and one study ${ }^{9}$ has

Accepted for publication 28 November 1983 shown the coexistence of acetylcholinesterase and catecholamines cytochemically in cultured murine neuroblastoma. Neuroblastoma now has recognised status among the neuroendocrine tumours, and one of the many characteristics of that tumour group is the ability to express acetylcholinesterase..$^{\circ}$ As far as we know, acetylcholinesterase activity has not been harnessed for clinical diagnosis of neuroblastoma, and the aim of the present study was to show by enzyme histochemistry its presence in two cases of human neuroblastoma. For comparison, the enzyme reaction was also evaluated in some other small cell tumours.

\section{Patients and methods}

One of the neuroblastomas was removed from a six month old baby girl who presented with an abdominal mass (case 1). Urinary concentration of vanilmandelic acid and homovanillic acid were increased and her blood pressure was raised. The second tumour was resected from the retroperitoneal region of a four month old baby boy, whose urine also contained a high concentration of vanilmandelic acid (case 2).

For electron microscopy, fresh tissue from both tumours was excised, diced into $1 \mathrm{~mm}$ cubes, and fixed in phosphate buffered $2 \%$ paraformaldehyde- $2.5 \%$ glutaraldehyde $\mathrm{pH} 7.3$ at $0-4^{\circ} \mathrm{C}$ (buffer osmolarity $288 \mathrm{mOsm} / \mathrm{l}$ ). In both cases the tissue was post-fixed in osmium tetroxide, processed by standard methods, and embedded in Araldite. 
Sections ( $1 \mu \mathrm{m}$ thick), stained with toluidine blue, were assessed to ensure representative sampling, and ultrathin sections, stained with uranyl acetate and lead citrate, were studied in an AEI Corinth 275 electron microscope.

Fresh tissue was also snap frozen in liquid nitrogen and stored (in liquid nitrogen) until required. Tissue was then sectioned in a cryostat at $10 \mu \mathrm{m}$ thickness and stained for acetylcholinesterase activity using a modification of the method of Karnovsky and Roots." "The remainder of the tumour was processed as for routine microscopy, embedded in paraffin wax, cut at $6 \mu \mathrm{m}$, and stained with haematoxylin and eosin.

The same histochemical procedure for acetylcholinesterase was also performed on rhabdomyosarcoma (four cases), lymphoblastic lymphoma (one case), and Ewing's tumour (one case). Of the four rhabdomyosarcomas, three conformed to the alveolar subtype; the other was of the embryonal pattern. The latter tumour was fairly well differentiated with many large rhabdomyoblasts and frequent cytoplasmic cross striations. The alveolar rhabdomyosarcomas were generally composed of small, undifferentiated cells with an occasional early rhabdomyoblast. Rare cytoplasmic cross striations were found in only one of these tumours.

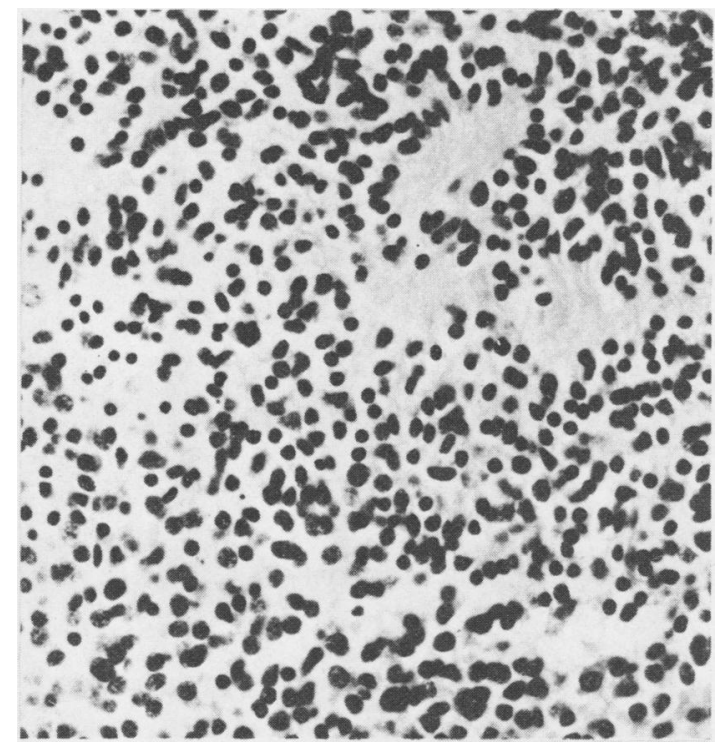

Fig. 1 Neuroblastoma from case 1 , showing an essentially undifferentiated small cell pattern but with several characteristic neurofibrillary rosettes. Haematoxylin and eosin. Original magnification $\times 145$.

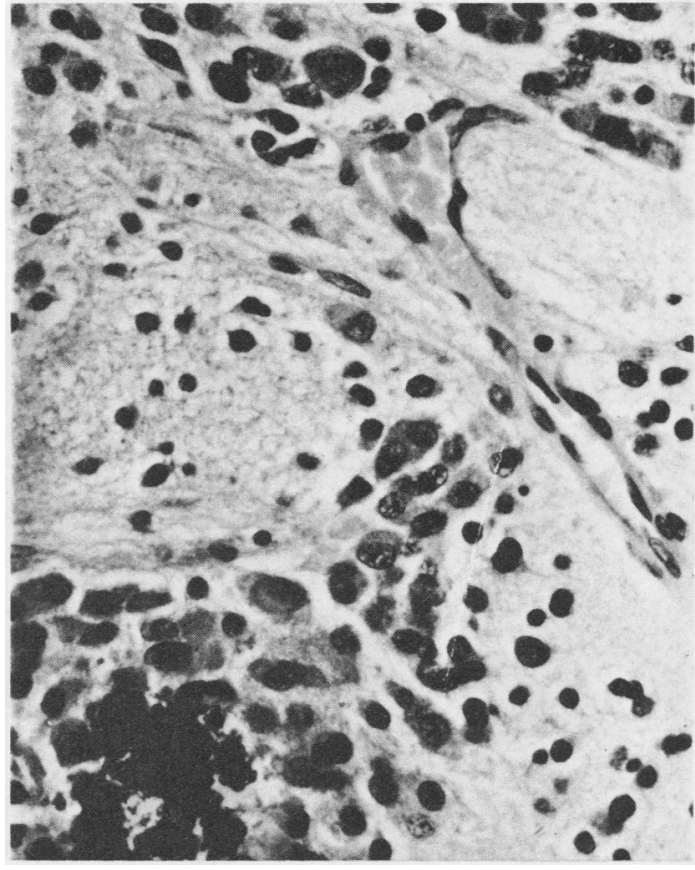

Fig. 2 Neuroblastoma from case 2, showing early ganglionic differentiation, patches of neurofibrillary material and focal calcification. Haematoxylin and eosin. Original magnification $\times 230$.

\section{Results}

Neuroblastoma was confirmed by conventional microscopy and electron microscopy in both tumours. In the tumour from case 1 this was generally poorly differentiated, but neurofibrillary rosettes, characteristic of neuroblastoma, were occasionally seen (Fig. 1). The tumour from case 2 showed moderate differentiation with focal calcification, early ganglionic differentiation, and patches of neurofibrillary matrix (Fig. 2). Both tumours showed variable acetylcholinesterase activity, which occurred as a brown granular precipitate mainly surrounding individual nuclei, which were pale by contrast (Fig. 3). Larger tumour cells seemed to show a more intense reaction (Fig. 4), but most of the smaller cells were also positive, albeit to a lesser degree. Anuclear patches of fine positive granularity, apparently corresponding to areas of neurofibrillary matrix seen in sections stained with haematoxylin and eosin, were also present. The centres of rosettes in the tumour from case 1 showed a similar reaction.

Cellular activity for acetylcholinesterase was present in three of the four rhabdomyosarcomas. This was conspicuous in the tumour with the embryonal pattern and prominent rhabdomyoblasts 


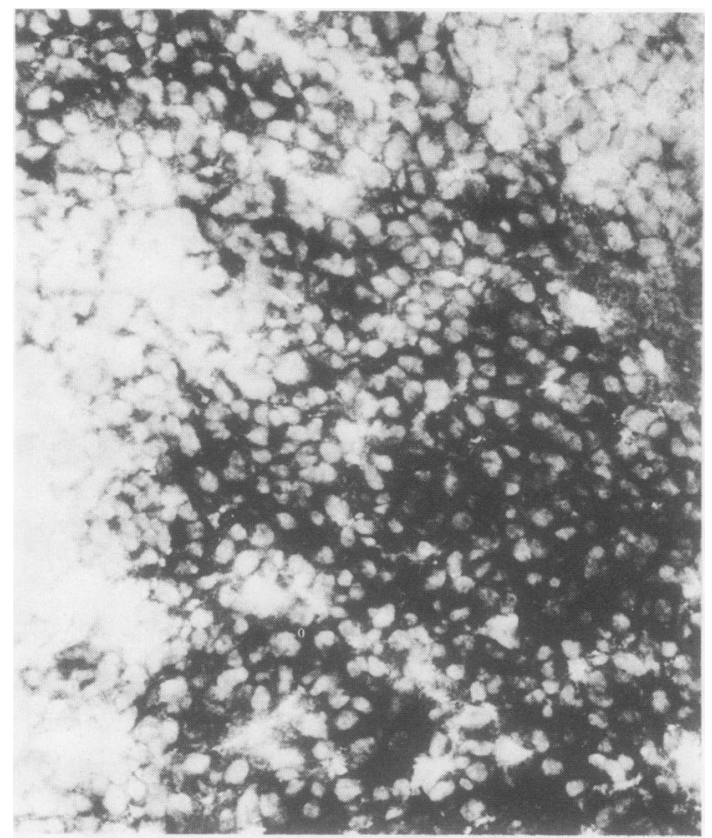

Fig. 3 Acetylcholinesterase in tumour from case 1, showing a generalised positive reaction with patchy accentuation. Acetylcholinesterase. Original magnification $\times 145$.

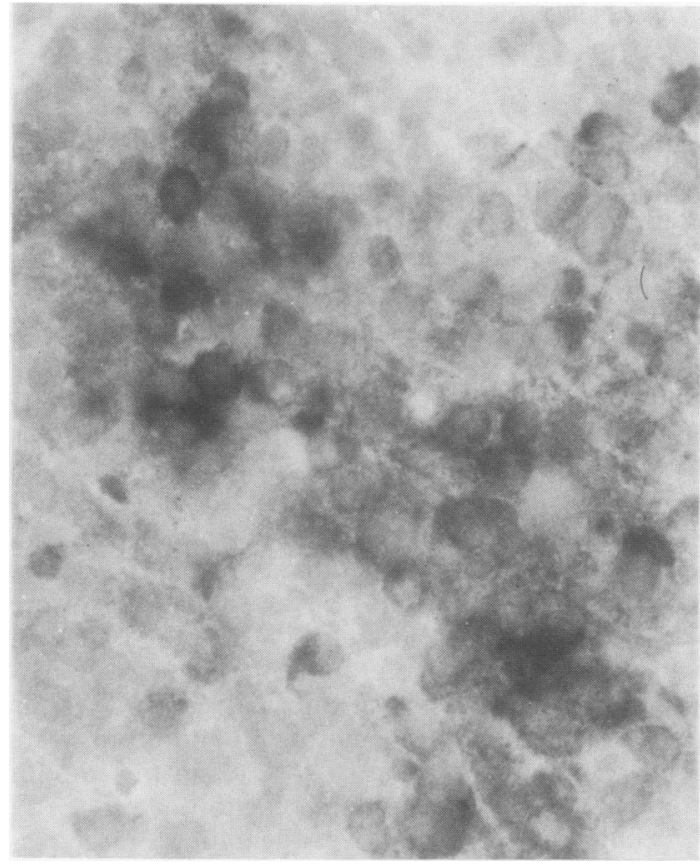

Fig. 4 Acetylcholinesterase in tumour from case 2, showing positive clumps of moderate sized cells corresponding to areas of early ganglionic differentiation. Acetylcholinesterase. Original magnification $\times 250$.
(Fig. 5). Two of the three tumours of the alveolar subtype showed positive staining, which was focal and sparse (Fig. 6). The remaining alveolar tumour was negative. The Ewing's tumour and lymphoma also showed no discernible reactivity for acetylcholinesterase.

Ultrastructurally, both neuroblastomas contained dense cored vesicles as well as small clear vesicles within their neurite processes. Cored vesicles generally measured $100-120 \mathrm{~nm}$ in diameter, being about twice the size of the clear vesicles (Fig. 7), which were $50-80 \mathrm{~nm}$ in diameter. Small clear vesicles were easily distinguishable from cross sectioned neurotubules, which measured $20 \mathrm{~nm}$ in diameter.

\section{Discussion}

Until 1972 it was generally assumed that neuroblastoma originated in sympathetic neurones and that catecholamine was its sole biochemical expression. Choline acetyltransferase, an acetylcholine synthesising enzyme, has since been shown biochemically in cultured murine neuroblastoma,${ }^{6}$ cultured human neuroblastoma, ${ }^{7}$ and also, more directly, in

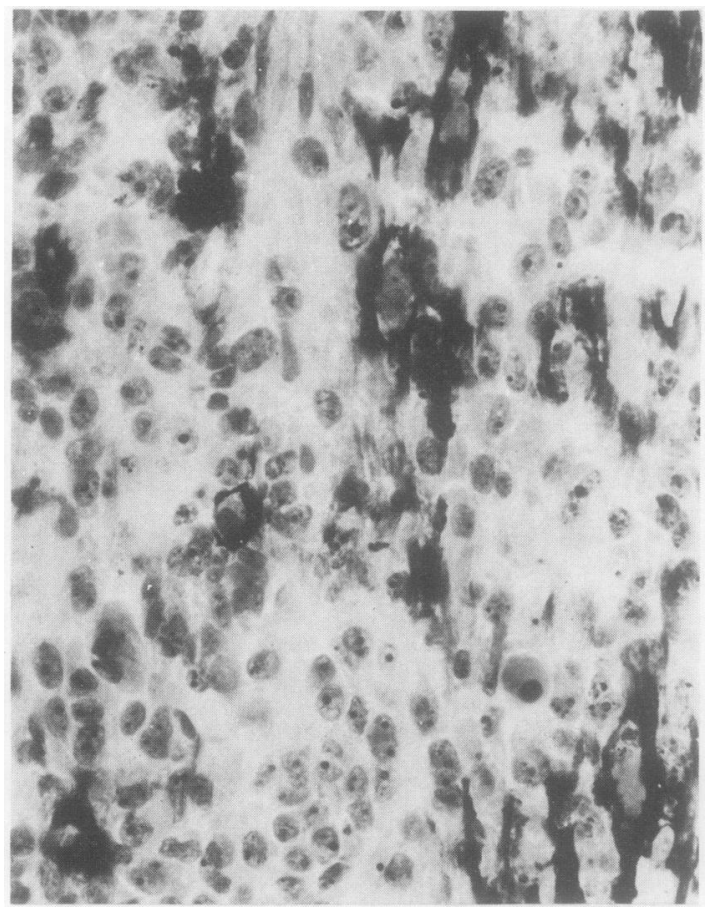

Fig. 5 Acetylcholinesterase in embryonal rhabdomyosarcoma showing conspicuous positivity, mainly in relation to large rhabdomyoblasts. The small, undifferentiated cells are generally negative.

Acetylcholinesterase. Original magnification $\times 330$. 


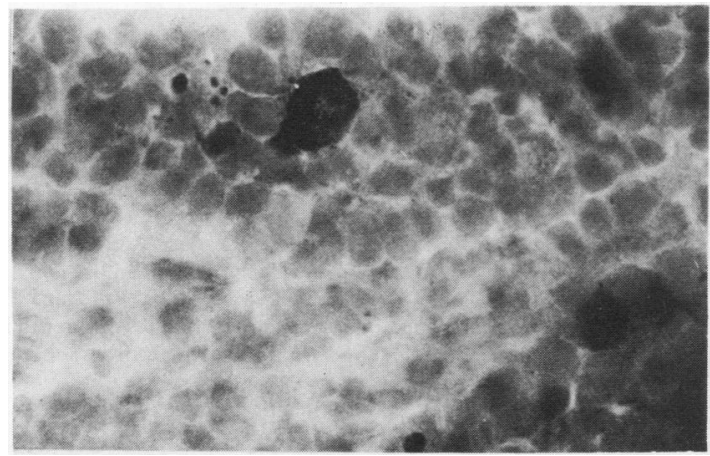

Fig. 6 Alveolar pattern of rhabdomyosarcoma with sparse focal acetylcholinesterase activity. Small rhabdomyoblasts were discernible only occasionally in routine stained sections. Acetylcholinesterase. Original magnification $\times 230$.

human tumour. ${ }^{8}$ Acetylcholinesterase also has been shown biochemically in cultured murine neuroblastoma, ${ }^{6}$ and one report ${ }^{9}$ has referred to its cytochemical demonstration in murine cultured neuroblas- toma. These investigations all support the biochemical diversity of the cell type of neuroblastoma. Acetylcholinesterase activity in our two randomly selected cases suggests that this is a common integral feature of this neoplasm. The combination of acetylcholinesterase activity, as shown here, and the clinical evidence in the two patients of catecholamine secretion, support further the existence of biochemical diversity of the neoplasm.

On the basis of the relative activity of two neurotransmitter synthesising enzymes, including choline acetyltransferase, three cell types in neuroblastoma have been defined. ${ }^{12}$ Choline acetyltransferase activity was variable and even absent in one tumour. In cultured murine tumour, acetylcholinesterase activity may be enhanced by restricting cell division ${ }^{13} 14$ or by adding acetylcholine to the culture medium. ${ }^{15}$ The former is apparently associated with concurrent morphological differentiation but the latter is independent of such differentiation. ${ }^{15}$ These experiments show that in vitro the enzyme is under the control of several factors. It is therefore difficult to predict the true incidence of histochemically demonstrable

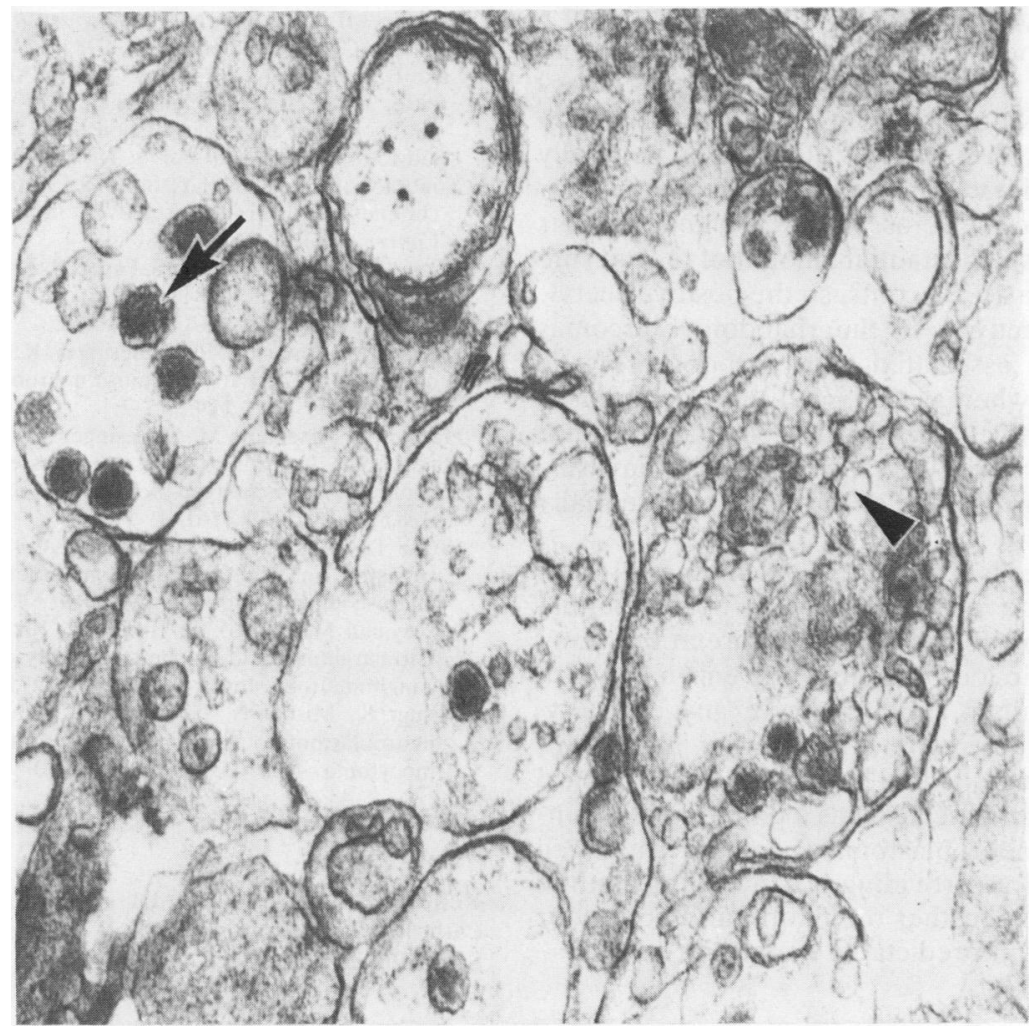

Fig. 7 Electron microscopic view of neurite processes in tumour from case 1, showing a mixture of dense cored vesicles (arrow) and clear vesicles (arrowhead). Uranyl acetate and lead citrate $\times 62500$. 
acetylcholinesterase activity in a larger sample of tumours.

Electron microscopy of both tumours showed three main types of intracytoplasmic vesicles: small cored vesicles, large cored vesicles, and small clear vesicles. Of these, only small cored vesicles have been shown to contain catecholamine, ${ }^{16}$ but Yokoyama et $\mathrm{al}^{17}$ could not exclude a similar function for the large cored vesicles in their ultrastructure study. While the additional presence of small clear vesicles has been described in neuroblastoma, no definite functional role seems to have been assigned to them. Small clear vesicles in axon profiles of mammalian tissue have been related to cholinergic neuronal transmission. ${ }^{5}$ It has been suggested that their presence in neuroblastoma represents the morphological aspect of a related function. ${ }^{18}$

The histochemical procedure for showing the presence of acetylcholinesterase is used widely in diagnosing Hirschsprung's disease, 1 and most laboratories dealing with childhood tissues are therefore already familiar with its application. The method is straightforward and can be executed in a few hours. Of the two tumours described here, one was moderately differentiated and, under ordinary circumstances, would present little difficulty with histological diagnosis. The other was generally poorly differentiated, however, and the demonstration of acetylcholinesterase activity in that tumour probably reflects a substantial amount of the enzyme in early neuroblasts. By contrast, the positive acetylcholinesterase activity in the rhabdomyosarcomas appeared to be associated with rhabdomyoblastic differentiation, whereas the small, undifferentiated cells were negative. This resulted in focal cellular positivity in two of the alveolar rhabdomyosarcomas, in which the cellular pattern was essentially undifferentiated.

Red blood cells contain acetylcholinesterase," and haemorrhage into tumours may be a potential source of concern for accurate interpretation; however, cytoplasmic localisation of the enzyme, which is easily discernible, should resolve any difficulty. Application of the method to a larger number of tumours will gauge its real value as a histochemical marker. The technique also has a potential to distinguish between neuroblastoma and other small cell tumours as well as providing a convenient method by which to study further the nature and behaviour of this clinically unpredictable neoplasm.

\section{References}

' Mackay B, Masse SR, King OY, Butler JJ. Diagnosis of neuroblastoma by electron microscopy of bone marrow aspirates. Pediatrics 1975;56:1045-9.

${ }^{2}$ Dhillon AP, Rode J, Leathern A. Neurone specific enolase: an aid to the diagnosis of melanoma and neuroblastoma. Histopathology 1982;6:81-92.

${ }^{3}$ Reynolds CP, Smith RG, Frenkel EP. The diagnostic dilemma of the "small round cell neoplasm". Cancer 1981;48:2088-94.

${ }^{4}$ Kemshead JT, Goldman A, Fritschy J, Malpas JS, Pritchard J. Use of panels of monoclonal antibodies in the differential diagnosis of neuroblastoma and lymphoblastic disorders. Lancet 1983; i: 12-14.

${ }^{5}$ Burnstock G. Ultrastructural identification of neurotransmitters. Scand J Gastroenterol 1981;16:1-9.

- Amano T, Richelson E, Nirenberg M. Neuroblastoma synthesis by neuroblastoma clones. Proc Natl Acad Sci 1972;69:258-63.

' Prasad KN, Mandal B, Kumar S. Demonstration of cholinergic cells in human neuroblastoma and ganglioneuroma. $J$ Pediatr 1973;82:677-9.

${ }^{8}$ Imashuky S, Inui A, Kusunoki T, Okabe H, Vonezawa T,

Miyake S. Acetylcholine synthesis in sympathetic human neuroblastoma. J Pediatr Surg 1976;11:949-54.

${ }^{9}$ Hermetet JC, Ciesielski-Treska J, Mandel P. Cytochemical demonstration of catecholamines and acetylcholinesterase activity in neuroblastoma cells in culture. $J$ Histochem Cytochem 1972;20:137-42.

${ }^{10}$ Pearse AGE. Neuroendocrine tumours and hyperplasia. In Filipe MI, Lake BD, eds. Histochemistry in pathology. Edinburgh: Churchill Livingstone, 1983:274-85.

" Lake BD, Puri P, Nixon HH, Claireaux AE. Hirschsprung's disease. An appraisal of histochemically demonstrated acetylcholinesterase activity in suction rectal biopsy specimens as an aid to diagnosis. Arch Pathol 1978;102:244-7.

12 Yokomori K, Tsuchido Y, Saito S. Tyrosine hydroxylase and choline acetyltransferase activity in human neuroblastoma Cancer 1983;52:263-72.

${ }^{13}$ Blume A, Gilbert F, Wilson S, Farber J, Rosenberg R, Nirenberg M. Regulation of acetylcholinesterase in neuroblastoma cells. Proc Nat Acad Sci 1970;67:786-92.

${ }^{14}$ Kates JR, Winterton R, Schlesinger K. Induction of acetylcholinesterase activity in mouse neuroblastoma tissue culture cells. Nature 1971;229:345-7.

is Harkins J, Arsenault M, Schlesinger K, Kates J. Induction of neuronal functions: Acetylcholinesterase-induced acetylcholinesterase activity in mouse neuroblastoma cells. Proc Nat Acad Sci 1972;69:3161-4.

${ }^{16}$ Wolfe DE, Potter LT, Richardson KC, Axelrod J. Localising tritriated norepinephrine in sympathetic axons by electron microscopic autoradiography. Science 1962;38:440.

17 Yokoyama M, Okado K, Tokue A, Takayasu H, Yamada R. Ultrastructural and biochemical study of neuroblastoma and ganglioneuroblastoma. Invest Urol 1971;9:156-64.

${ }^{18}$ Misugi K, Misugi N, Newton WA. Fine structural study of neuroblastoma, ganglioneuroblastoma and pheochromocytoma. Arch Pathol 1968;86:160-9.

Request for reprints to: Dr S Variend, Department of Pathology, Children's Hospital, Western Bank, Sheffield S10 2TH, England. 